Modern Physics Letters A

(C) World Scientific Publishing Company

\title{
Observation of Galactic Sources of Very High Energy $\gamma$-Rays with the MAGIC Telescope
}

\author{
H. BARTKO for the MAGIC collaboration \\ MPI für Physik, Werner-Heisenberg-Institut, \\ Föhringer Ring 6, D-80805 München, Germany \\ E-mail: hbartko@mppmu.mpg.de
}

Received (Day Month Year)

Revised (Day Month Year)

\begin{abstract}
The MAGIC telescope with its $17 \mathrm{~m}$ diameter mirror is today the largest operating singledish Imaging Air Cherenkov Telescope (IACT). It is located on the Canary Island La Palma, at an altitude of $2200 \mathrm{~m}$ above sea level, as part of the Roque de los Muchachos European Northern Observatory. The MAGIC telescope detects celestial very high energy $\gamma$-radiation in the energy band between about $50 \mathrm{GeV}$ and $10 \mathrm{TeV}$. Since the autumn of 2004 MAGIC has been taking data routinely, observing various objects, like supernova remnants (SNRs), $\gamma$-ray binaries, Pulsars, Active Galactic Nuclei (AGN) and Gamma-ray Bursts (GRB). We briefly describe the observational strategy, the procedure implemented for the data analysis, and discuss the results of observations of Galactic Sources.
\end{abstract}

Keywords: $\mathrm{TeV} \gamma$-ray astrophysics - super nova remnants, pulsars, binary systems

PACS Nos.: 95.85.Pw, 95.85.Ry

\section{Introduction - The MAGIC Telescope}

MAGIC $16 \mid 26$ is currently the largest single dish Imaging Air Cherenkov Telescope (IACT) in operation. Located on the Canary Island La Palma $\left(28.8^{\circ} \mathrm{N}, 17.8^{\circ} \mathrm{W}\right.$, $2200 \mathrm{~m}$ a.s.l.), it has a $17-\mathrm{m}$ diameter tessellated parabolic mirror, supported by a light weight carbon fiber frame. It is equipped with a high quantum efficiency 576pixel $3.5^{\circ}$ field-of-view photomultiplier camera. The analog signals are transported via optical fibers to the trigger electronics and the 2 GSamples/s FADC system 18 .

The MAGIC telescope can operate under moderate moonlight or twilight conditions 9 . For these conditions, no change in the high voltage settings is necessary as the camera PMTs were especially designed to avoid high currents.

The physics program of the MAGIC telescope includes both, topics of fundamental physics and astrophysics. In this paper the observations of galactic sources are reviewed. The observations of extragalactic sources are presented elsewhere, see e.g. 30 . 


\section{Data Analysis}

The data analysis is generally carried out using the standard MAGIC analysis and reconstruction software 23 , the first step of which involves the FADC signal reconstruction and the calibration of the raw data 34/7. After calibration, image-cleaning tail cuts are applied (see e.g. 31). The camera images are parameterized by image parameters 38 . The Random Forest method (see $21 \mid 22$ for a detailed description) was applied for the $\gamma /$ hadron separation (for a review see e.g. ${ }^{31}$ ) and the energy estimation.

For each event, the arrival direction of the primary $\gamma$-ray candidate in sky coordinates is estimated using the DISP-method resulting in VHE $\gamma$-ray sky maps 32 43329. The angular resolution of this procedure is $\sim 0.1^{\circ}$, while the source localization in the sky is provided with a systematic error of $1^{\prime} 11$.

The differential VHE $\gamma$-ray spectrum $\left(\mathrm{dN}_{\gamma} /\left(\mathrm{dE}_{\gamma} \mathrm{dAdt}\right)\right.$ vs. true $\left.\mathrm{E}_{\gamma}\right)$ is corrected (unfolded) for the instrumental energy resolution 13|20|12. All fits to the spectral points take into account the correlations between the spectral points that are introduced by the unfolding procedure.

The systematic error in the flux level determination depends on the slope of the $\gamma$-ray spectrum. It is typically estimated to be $35 \%$ and the systematic error in the the spectral index is 0.2 411].

\section{Galactic Sources}

The observations with the MAGIC telescope included the following types of objects: VHE $\gamma$-ray sources coincident with supernova remnants (section 3.1), the Galactic Center (section 3.2), the $\gamma$-ray binary LS I +61 303 (section 3.3), pulsars and pulsar wind nebulae (section 3.4).

\subsection{Unidentified $\mathrm{VHE} \gamma$-ray sources coincident with Supernova remnants}

Shocks produced by supernova explosions are assumed to be the source of the galactic component of the cosmic ray flux 15. In inelastic collisions of high energy cosmic rays with ambient matter $\gamma$-rays and neutrinos are produced. These neutral particles give direct information about their source, as their trajectories are not affected by the Galactic and extra Galactic magnetic fields in contrast to the charged cosmic rays. However, not all VHE $\gamma$-rays from galactic sources are due to the interactions of cosmic rays with ambient matter. There are also other mechanisms for the production of VHE $\gamma$-rays like the inverse Compton up-scattering of ambient low energy photons by VHE electrons. For each individual source of VHE $\gamma$-rays, the physical processes of particle acceleration and $\gamma$-ray emission in this source have to be determined. A powerful tool is the modeling of the multiwavelength emission of the source taking into account the ambient gas density as traced by CO observations 47.

Within its program of observation of galactic sources, MAGIC has taken data on 
a number of supernova remnants, resulting in the discovery of VHE $\gamma$-ray emission from a source in the SNR IC443, MAGIC J0616+225 10. Moreover, two recently discovered VHE $\gamma$-ray sources, which are spatially coincident with SNRs, HESS J1813178 and HESS J1834-087 ${ }^{2}$ have been observed with the MAGIC telescope 316 .

IC443 is a well-studied shell-type SNR near the Galactic Plane with a diameter of $45^{\prime}$ at a distance of about $1.5 \mathrm{kpc}$. It is a prominent source and it has been studied from radio waves to $\gamma$-rays of energies around $1 \mathrm{GeV}$. 3317 and 40 extrapolated the energy spectrum of 3EG J0617+2238 into the VHE $\gamma$-ray range and predicted readily observable fluxes. Nevertheless, previous generation IACTs have only reported upper limits to the VHE $\gamma$-ray emission 41139 . The observation of IC 443 using the MAGIC Telescope has led to the discovery of a new source of VHE $\gamma$-rays, MAGIC J0616+225. The flux level of MAGIC J0616+225 is lower and the energy spectrum (fitted with a power law of slope $\Gamma=-3.1 \pm 0.3$ ) is softer than the predictions. The coincidence of the VHE $\gamma$-ray source with SNR IC 443 suggests this $\mathrm{SNR}$ as a natural counterpart. A massive molecular cloud and $\mathrm{OH}$ maser emissions are located at the same sky position as that of MAGIC J0616+225, see figure 2. This suggests that a hadronic origin of the VHE $\gamma$-rays is possible. However, other mechanisms for the VHE $\gamma$-ray emission cannot be excluded yet.

HESS J1834-087 is spatially coincident with the SNR G23.3-0.3 (W41). W41 is an asymmetric shell-type SNR, with a diameter of $27^{\prime}$ at a distance of $\sim 5 \mathrm{kpc}$. It is a prominent radio source, and only recently 42 found a faint X-ray source within the area of W41 in data from the Swift satellite and 46 found an extended $\mathrm{X}$-ray feature spatially coincident with the VHE $\gamma$-ray emission. As in the case of IC 443, the VHE $\gamma$-radiation of W41 is associated with a large molecular complex called "[23,78]" 27, see figure 1. Although the mechanism responsible for the VHE $\gamma$-radiation has not yet been clearly identified, it could be produced by high energy hadrons interacting with the molecular cloud.

HESS J1813-178 is spatially coincident with SNR G12.8-0.0 with a diameter of 2 ' at a distance of $\sim 4 \mathrm{kpc}$. It exhibits relatively faint radio and X-radiation. This source is also located in a relatively high-density environment? 37 discovered a putative pulsar wind nebula and associated it with the VHE $\gamma$-ray source.

These results confirm that Galactic VHE $\gamma$-ray sources are usually spatially correlated with SNRs. Nevertheless, the exact nature of the parent particles of the VHE $\gamma$-rays, their acceleration (in SNR shocks or PWN), and the processes of $\gamma$-ray emission need (or still require) further study.

\subsection{The Galactic Center}

The Galactic Center region contains many remarkable objects which may be responsible for high-energy processes generating $\gamma$-rays: A super-massive black hole, supernova remnants, candidate pulsar wind nebulae, a high density of cosmic rays, hot gas and large magnetic fields. Moreover, the Galactic Center may appear as the brightest VHE $\gamma$-ray source from the annihilation of possible dark matter particles 


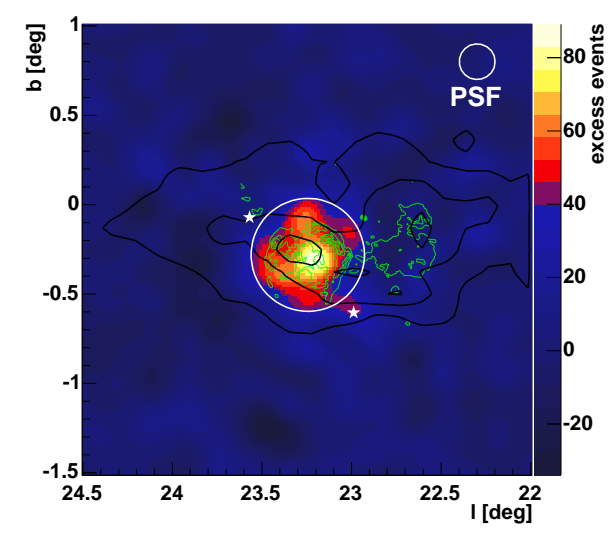

Fig. 1. Sky map of $\gamma$-ray candidate events (background subtracted) in the direction of HESS J1834-087 for an energy threshold of about $250 \mathrm{GeV}$. The source is clearly extended with respect to the MAGIC PSF (small white circle). The two white stars denote the tracking positions of the MAGIC telescope. Overlayed are ${ }^{12} \mathrm{CO}$ emission contours (black) from ${ }^{28}$ and contours of $90 \mathrm{~cm}$ VLA radio data from 49 (green). The ${ }^{12} \mathrm{CO}$ contours are at $25 / 50 / 75 \mathrm{~K} \mathrm{~km} / \mathrm{s}$, integrated from 70 to 85 $\mathrm{km} / \mathrm{s}$ in velocity, the range that best defines the molecular cloud associated with W41. The contours of the radio emission are at $0.04 / 0.19 / 0.34 / 0.49 / 0.64 / 0.79$ Jy/beam, chosen for best showing both SNRs G22.7-0.2 and G23.3-0.3 at the same time. Clearly, there is no superposition with SNR G22.7-0.2. The central white circle denotes the source region integrated for the spectral analysis. ${ }^{6}$.

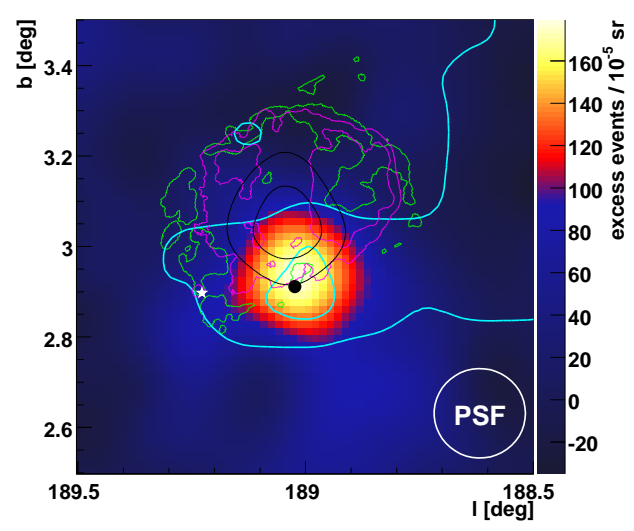

Fig. 2. Sky map of $\gamma$-ray candidate events (background subtracted) in the direction of MAGIC J0616+225 for an energy threshold of about $150 \mathrm{GeV}$. The cyan ${ }^{12} \mathrm{CO}$ contours ${ }^{28}$ are at 7 and $14 \mathrm{~K} \mathrm{~km} / \mathrm{s}$, integrated from -20 to $20 \mathrm{~km} / \mathrm{s}$ in velocity, the range that best defines the molecular cloud associated with IC 443. The green contours of $20 \mathrm{~cm}$ VLA radio data ${ }^{25}$ are at $5 \mathrm{mJy} /$ beam, chosen for best showing both the SNR IC 443. The purple Rosat X-ray contours 14 are at 700 and 1200 counts / $6 \cdot 10^{-7}$ sr. The black EGRET contours ${ }^{36}$ represent a $68 \%$ and $95 \%$ statistical probability that a single source lies within the given contour. The white star denotes the position of the pulsar CXOU J061705.3+222127 ${ }^{45}$. The black dot shows the position of the $1720 \mathrm{MHz} \mathrm{OH}$ maser 24 . The white circle shows the MAGIC PSF of $\sigma=0.1^{\circ} .{ }^{10}$.

19 of all proposed dark matter particle annihilation sources.

The Galactic Center was observed with the MAGIC telescope 4 under large zenith angles, resulting in the measurement of a differential $\gamma$-ray flux, consistent with a steady, hard-slope power law between $500 \mathrm{GeV}$ and about $20 \mathrm{TeV}$, with a spectral index of $\Gamma=-2.2 \pm 0.2$. This result confirms the previous measurements by the HESS collaboration. The VHE $\gamma$-ray emission does not show any significant time variability; the MAGIC measurements rather affirm a steady emission of $\gamma$-rays from the GC region on time scales of up to one year.

The VHE $\gamma$-ray source is centered at $(\mathrm{RA}, \mathrm{Dec})=\left(17^{\mathrm{h}} 45^{\mathrm{m}} 20^{\mathrm{s}},-29^{\circ} 2^{\prime}\right)$. The excess is point-like, its location is consistent with SgrA*, the candidate PWN G359.95-0.04 as well as SgrA East. The nature of the source of the VHE $\gamma$-rays has not yet been (or yet to be) identified. The power law spectrum up to about $20 \mathrm{TeV}$ disfavours 


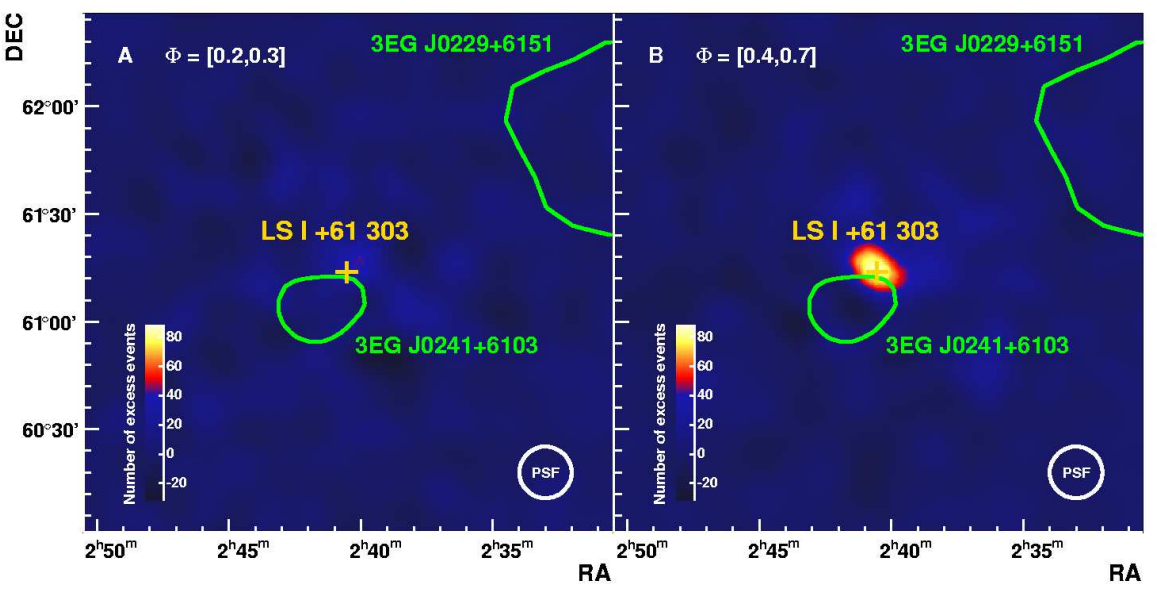

Fig. 3. Smoothed maps of $\gamma$-ray excess events above $400 \mathrm{GeV}$ around LS I +61303 . (A) 15.5 hours corresponding to data around periastron, i.e. between orbital phases 0.2 and 0.3. (B) 10.7 hours at orbital phase between 0.4 and 0.7. The number of events is normalized in both cases to 10.7 hours of observation. The position of the optical source LSI +61 303 (yellow cross) and the 95\% confidence level contours for 3EG J0229+6151 and 3 EG J0241+6103 (green contours) ${ }^{36}$, are also shown. The bottom-right circle shows the size of the point spread function of MAGIC ( $1 \sigma$ radius). No significant excess in the number of $\gamma$-ray events is detected around periastron passage, while it shows up clearly (9.4 $\sigma$ statistical significance) at later orbital phases, in the location of LS I $+61303 .{ }^{5}$.

dark matter annihilation as the main origin of the detected flux. The absence of flux variation indicates that the VHE $\gamma$-rays are rather produced in a steady object such as a SNR or a PWN, and not in the central black hole.

\subsection{The $\gamma$-ray binary $L S I+61303$}

This $\gamma$-ray binary system is composed of a B0 main sequence star with a circumstellar disc, i.e. a Be star, located at a distance of

$\sim 2 \mathrm{kpc}$. A compact object of unknown nature (neutron star or black hole) is orbiting around it, in a highly eccentric $(e=0.72 \pm 0.15)$ orbit.

LS I +61 303 was observed with MAGIC for 54 hours between October 2005 and March 2006 . The reconstructed $\gamma$-ray sky map is shown in figure 3. The data were first divided into two different samples, around periastron passage $(0.2-0.3)$ and at higher (0.4-0.7) orbital phases. No significant excess in the number of $\gamma$ ray events is detected around periastron passage, whereas there is a clear detection (9.4 $\sigma$ statistical significance) at later orbital phases. Two different scenarios were discussed to explain this high energy emissions: the microquasar scenario where the $\gamma$-rays are produced in a radio-emitting jet; or the pulsar binary scenario, where they are produced in the shock which is generated by the interaction of a pulsar 


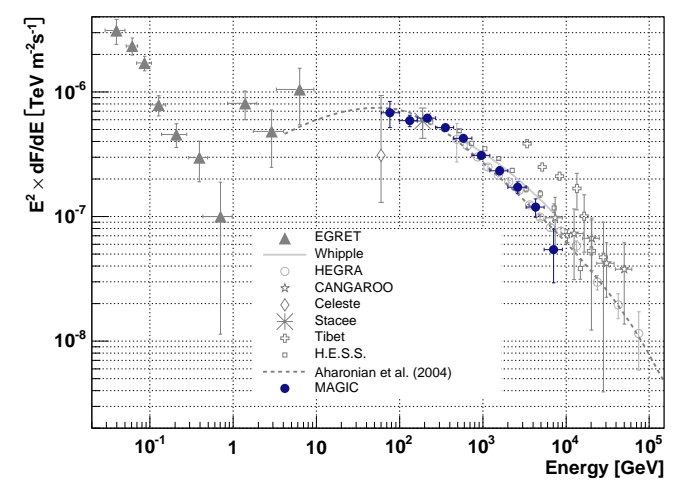

Fig. 4. Spectral energy distribution of the $\gamma$-ray emission of the Crab Nebula. The measurements below $10 \mathrm{GeV}$ are from the EGRET, the measurements above are from groundbased experiments. Above $400 \mathrm{GeV}$ the MAGIC data are in agreement with measurements of other IACTs. The dashed line represents a model prediction by ${ }^{1}{ }^{11}$.

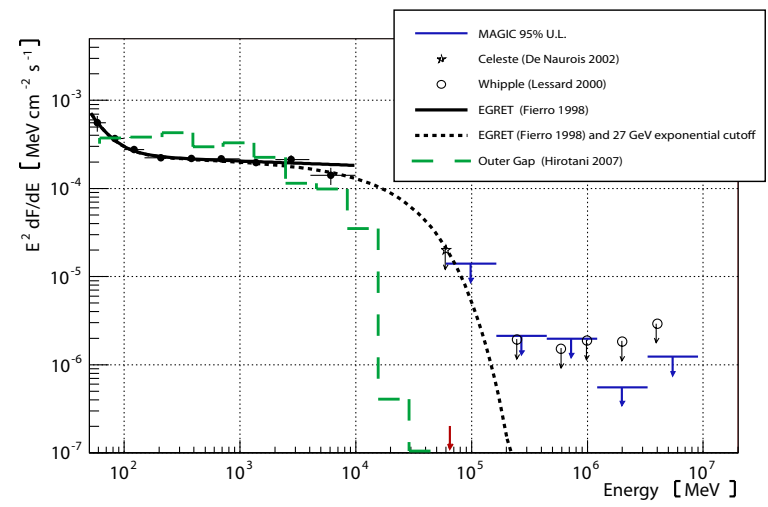

Fig. 5. Upper limits (95\% CL.) on the pulsed $\gamma$-ray flux from the Crab Pulsar; upper limits in bins of energy are given by the blue points. The upper limit on the cutoff energy of the pulsed emission is indicated by the dashed line. The analysis threshold to derive the upper limit on the cutoff energy is indicated by the red arrow. ${ }^{11}$.

wind and the wind of the massive companion.

\subsection{Pulsars and Pulsar Wind Nebulae}

The Crab Nebula is a bright and steady emitter of $\mathrm{GeV}$ and $\mathrm{TeV}$ energies, and is therefore an excellent calibration candle. This object has been observed intensively in the past, over a wide range of wavelengths.

The energy domain between 10 and $100 \mathrm{GeV}$ is of particular interest, as both the Inverse Compton peak of the spectral energy distribution and the cut-off of the pulsed emission is expected in this energy range.

A significant amount of MAGIC's observation time has been devoted to observ- 
ing the Crab Nebula, both for technical (because it is a strong and steady emitter) and astrophysical studies. A sample of 16 hours of selected data has been used to measure the energy spectrum between $60 \mathrm{GeV}$ and $9 \mathrm{TeV}$, and the result is shown in figure 4 11. Also, a search for pulsed $\gamma$-ray emission from the Crab Pulsar has been carried out. Figure 5 shows the derived (95\% CL.) upper limits.

Pulsed $\gamma$-ray emission was also searched for from the pulsar PSR B1951+32. A $95 \%$ CL. of $4.3 \cdot 10^{-11} \mathrm{~cm}^{-} 2 \mathrm{sec}^{-1}$ was obtained for the flux of pulsed $\gamma$-ray emission for $E_{\gamma}>75 \mathrm{GeV}$ and of $1.5 \cdot 10^{-11} \mathrm{~cm}^{-} 2 \mathrm{sec}^{-1}$ for the steady emission for $E_{\gamma}>140 \mathrm{GeV}$.

\section{Acknowledgments}

We thank the IAC for the excellent working conditions at the ORM in La Palma. The support of the German BMBF and MPG, the Italian INFN, the Spanish CICYT is gratefully acknowledged. This work was also supported by ETH research grant TH-34/04-3, and the Polish MNiI grant 1P03D01028.

\section{References}

1. Aharonian, et al. 2004, ApJ, 614, 897.

2. Aharonian, F. et al. (HESS Collab.), 2006, ApJ, 636, 777.

3. Albert, J. et al. (MAGIC Collab.), 2006, ApJ, 637, L41.

4. Albert, J. et al. (MAGIC Collab.), 2006, ApJ, 638, L101.

5. Albert, J. et al. (MAGIC Collab.), 2006, Science 312, 1771.

6. Albert, J. et al. (MAGIC Collab.), 2006, ApJ, 643, L53.

7. Albert, J. et al. (MAGIC Collab.), 2006, NIM submitted, astro-ph/0612385.

8. Albert, J. et al. (MAGIC Collab.), 2007, ApJ submitted, astro-ph/0702077.

9. Albert, J. et al. (MAGIC Collab.), 2007, Astropart. Phys. submitted, astro-ph/0702475.

10. Albert, J. et al. (MAGIC Collab.), 2007, ApJL submitted, arXiv:0705.3119.

11. Albert, J. et al. (MAGIC Collab.), 2007, ApJ submitted, arXiv:0705.3244.

12. Albert, J. et al. (MAGIC Collab.), 2007, NIM submitted, arXiv:0707.2453

13. Anykeev, V. B., Spiridonov, A. A. \& Zhigunov, V.B., 1991, NIM, A303, 350.

14. Asaoka, I. \& Aschenbach, B., 1994, A\&A, 284, 573.

15. Baade, W. \& Zwicky, F., 1934, Phys. Rev., 46, 76.

16. Baixeras, C. et al., 2004, NIM, A518, 188.

17. Baring, M. G. et al., 1999, ApJ, 513, 311.

18. Bartko, H. et al., 2005, NIM, A548, 464.

19. Bartko, H. et al. (MAGIC Collab.), Proc. 29th ICRC, Pune, India, 4-17, astro-ph/0508273

20. Bertero, M., 1989, Adv. Elect. and Elect. Phys., 75, 1.

21. Bock, R. K. et al., 2004, NIM, A516, 511.

22. Breiman, L., 2001, Machine Learning, 45, 5.

23. Bretz, T. \& R. Wagner (MAGIC Collab.), 2003, Proc. 28th ICRC, Tsukuba, Japan, 2947.

24. Claussen, M. J. et al., 1997, ApJ, 489, 143.

25. Condon, J. J. et al., 1998, AJ, 115, 1693.

26. Cortina, J. et al. (MAGIC Collab.), 2005, Proc. 29th ICRC, Pune, India, 5-359. 
27. Dame, T. M. et al., 1986, ApJ 305, 892.

28. Dame, T. M., Hartmann, D. \& Thaddeus, P., 2001, ApJ, 547, 792.

29. Domingo-Santamaria, E. et al. (MAGIC Collab.), 2005, Proc. 29th ICRC, Pune, India, 5-363, astro-ph/0508274

30. Errando, M., 2006, astro-ph/0701202.

31. Fegan, D. J., 1997, J Phys G, 23, 1013.

32. Fomin, V. P. et al., 1994, Astroparticle Physics, 2, 137.

33. Gaisser, T. K., Protheroe, R. J. \& Stanev, T. 1998, ApJ, 492, 219.

34. Gaug, M. et al. (MAGIC Collab.), 2005, Proc. 29th ICRC, Pune, India, 5-375, astro-ph/0508274.

35. Green, D. A., 2004, BASI, 32, 335G.

36. Hartman, R. C., 1999, ApJS, 123, 79.

37. Helfand, D.J. et al., 2007, ApJ, submitted (arXiv:0705.0065)

38. Hillas, A. M., 1985, Proc. 19th ICRC, La Jolla, 3, 445.

39. Holder, J. et al., 2006, AIP Conf. Proc., 745, 275.

40. Kaul, R. K., 2001, BASI, 29, 371.

41. Khelifi, $\quad$ B., 2003, Ph.D. thesis, available at http://lpnp90.in2p3.fr/\%7Ecat/Thesis/khelifi.pdf.

42. Landi, R. et al., 2006, ApJ, 651, 190.

43. Lessard, R. W. et al., 2001, Astroparticle Physics, 15, 1.

44. Majumdar, P. et al. (MAGIC Collab.), 2005, Proc. 29th ICRC, Pune, India, 5-203.

45. Olbert, Ch. M. et al., 2001, ApJ, 554, L205.

46. Tian, W. W. et al., 2007, ApJ 657, L25

47. Torres, D. F. et al., 2003, Phys. Rept., 382, 303.

48. Torres, D. F., 2004, ApJ, 617, 966.

49. White, R. L., Becker, R. H., \& Helfand, D. J., 2005, AJ, 130, 586. 